\title{
Chemical burns in children - evolution of cases over the course of the past years
}

\author{
Laura VASILE ${ }^{1}$, Raluca TATAR ${ }^{1,2}$, Dan Mircea ENESCU ${ }^{1,2}$ \\ 1"Grigore Alexandrescu” Emergency Clinical Hospital, Bucharest, Romania \\ 2"Carol Davila" University of Medicine and Pharmacy, Bucharest, Romania
}

\begin{abstract}
Burns, regardless of aetiology, are a common cause of injuries at pediatric ages, and these account for a large proportion of total burns. The frequency of chemical burns in children is relatively low in comparison with the overall amount of burns occurring at that age.

The present study follows the evolution of the total number of patients who met the criteria for a burn diagnosis, hospitalized over the course of 7 years, between 2012 and 2018, at "Grigore Alexandrescu" Emergency Clinical Hospital, as well as the evolution of patients with chemical burns from the same time period, as it is of ut-most importance to establish the evolutionary trend of the number of hospital visits and admissions, taking into consideration the well documented and established economic and psychological impact which a burn has on an individual. The results of the study reveal that the number of patients with chemical burns is increasing, however the necessity for their hospitalization is decreasing.
\end{abstract}

Keywords: burn, children, accident, prevention

\section{INTRODUCTION}

Burns, regardless of aetiology, are a common cause of accidents in pediatric ages. It is established that 40$50 \%$ of all burns consist of children's burns.

Burns can be classified by aetiological agent: thermic burns (flame, hot liquids, explosion, hot solids), chemical burns, electrical burns, irradiation $(1,2)$.

In the case of chemical burns, the aetiological agent consists of a chemical substance, which has the capacity of causing coagulative necrosis of tissue proteins, the local lesions being similar to those caused by a thermal burn $(3,4)$.

Chemical burns have a low incidence in our country, which is why we sometimes have the tendency to neglect this pathology, since we do not habitually encounter it in daily practice.

Approximately 25,000 products are involved in the emergence of chemical burns, which are predominantly found in the daily activity within sectors such as agriculture, industrial activity, the army, as well as in domestic use (in particular cleaning products) (5).

The number of chemical burns compared to the rest of the burns of different aetiology, is relatively low, accounting for approximately $10 \%$ of all burns, according to a study conducted in England between 2003 and 2011, but the mortality rate of these accidents is significantly high, accounting for approximately $30 \%$ of the total of patients who were declared deceased due to sustained burns $(1,2)$.

Chemical substances, acting as aetiological agents, which are involved in the production of chemical burns, present a high degree of variability, the most common being those in the class of acids and bases. The production mechanism of burns is particularly complex, being different from one substance to another:

- acids (hydrochloric acid, sulphuric acid, nitric acid, phosphoric acid etc.) cause exothermic reactions, producing coagulated necrosis, while 
the proteins are being decomposed to amino acids. In addition to this, the mechanism of action of acids consists of desiccation, as well as calcium chelation.

- the bases (sodium hydroxide, potassium hydroxide, etc.) produce a protein hydrolysis reaction in the tissues with which they come into contact $(3,4)$.

Based on the mechanism of action, the chemical substances which can produce lesions are:

- reducing substances - act by reducing peptide bonds, exotherm reaction

- oxidizing substances - act by adding an atom of oxygen, sulfur or halogen to the structure of proteins, which alters their functionality (sodium hypochlorite, potassium permanganate, peroxides, chromic acid)

- corrosive substances - corrode the skin and produce massive protein distortions (phenols, sodium hydroxide, potassium, ammonium and calcium)

- plasmatic toxic substances - form esters with proteins or inhibit inorganic ions, which are necessary for normal cellular function (formic, acetic, oxalic, hydrofluoric acid)

- desiccants - hygroscopic agents, which extract water from tissues, in the context of exothermic reactions (concentrated sulphuric acid)

- blistering substances - act by alkylating the DNA; produce vesicles, as a result of the release of proteases from the lysosomes of altered basal cells (1-3).

In the particular case of chemical burns, the skin lesion appears as a result of the action of heat released locally, the rapid dehydration of tissues with which the chemical comes into contact, the necrotic action of the chemical, the action of saponification, pertaining to fatty substances (1-4).

In order to increase the complexity of a chemical burn, in addition to local lesions, there are also phenomena of systemic intoxication (kidney, liver, pulmonary lesions), especially in the case of burns produced by acids - acetic acid, carbonic acid, oxalic acid, the chemical being absorbed into the systemic circulation at the level of the skin lesion.

The therapeutic process is continuous and lasts throughout the evolution of the burn, from the moment of the occurrence of the triggering event, until the moment of complete healing of the burn. The speed and accuracy of the treatment have the potential to make major differences in the subsequent evolution of the patient. The medical care of the burn victim begins at the scene of the accident, and is continued in the hospital, the treatment having both a local, skin component, as well as a general, systemic component $(3,4,6,7)$.

Studies conducted on adult patients, in whom chemical burns occur during technological processes involving chemicals having an increased concentration, affecting a large body surface, suggest that in most cases, first intention surgical treatment - excision-grafting is needed. In the case of children, the majority of these accidents occur in the domestic environment, where the concentration of the aetiological agent is usually much lower, the affected body surface and the depth of the injuries produced are limited, so it must be determined whether the patient can be treated in an outpatient regime or not, and in the case of hospitalization, therapeutic management of the case should be decided upon, both locally, as well as on a systemic level $(3,4,6)$.

To date, a unique therapeutic protocol or an ideal local and systemic treatment for chemical burns has yet to be developed, as these depend on a high number of variables (water-electrolyte imbalances, renal, hepatic, cardiac damage), which can sometimes change over the course of the healing process, taking into account the fact that the human body is constantly adapting to environmental factors.

\section{OBJECTIVES OF THE STUDY}

It is well-known that the incidence of chemical burns in children is relatively low compared to the total number of burns which occur at this age. The present study follows the evolution of the total number of patients who met the criteria for a burn diagnosis, their hospital visits and subsequent hospitalisation over the span of 7 years, between 2012 and 2018, at "Grigore Alexandres$\mathrm{cu}^{\prime}$ "Emergency Clinical Hospital, as well as the evolution of patients with chemical burns, as it is of utmost importance to establish the evolutionary trend of the number of hospital visits and hospitalisations, in view of the documented economic and psychological impact which a burn has on a person.

\section{MATERIAL AND METHODS}

A retrospective study was carried out, comparing the total number of burn patients who arrived at the emergency room (ER) of "Grigore Alexandrescu" Emergency Clinical Hospital and were subsequently hospitalised during this period, with the total number of patients with chemical burns who arrived and were hospitalised in this clinic.

\section{RESULTS}

Thus, between 01.01.2012 and 31.12.2018, within the Plastic Surgery and Burns Clinic of "Grigore Alexan- 
drescu" Emergency Clinical Hospital, a total number of 6915 patients with burn injuries arrived, of which 106 patients suffered burns produced by a chemical agent, amounting to $1.5 \%$ of the total number of hospital visits - Figure 1.

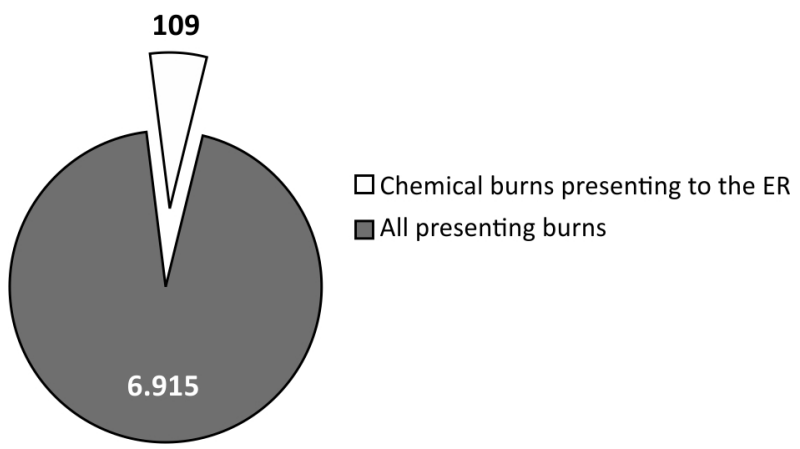

FIGURE 1. Distribution of cases arrived in the EAU (emergency accident unit) for chemical burns versus total number of hospital visits for burns, accumulated over the period 20122018

As well as this, the total number of hospitalisations during the same period was monitored, with 3,101 patients with burns admitted to the the Plastic Surgery and Burns Clinic being recorded, of which 14 patients are hospitalised with the diagnosis of chemical burn, these patients amounting to approximately $0.5 \%$ of the total number of patients who required hospitalisation - Figure 2.

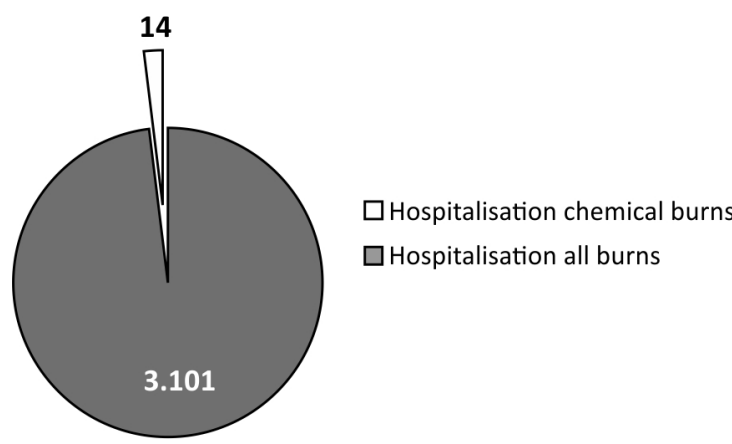

FIGURE 2. Distribution of hospitalisations for chemical burns versus total number of hospital visits due to burns, accumulated over the period 2012-2018

The analysis of the total number of patients with burns who arrived at the emergency room of the "Grigore Alexandrescu" Hospital over the course of the 7 years, when the study was conducted, indicates that in the first 2 years, the number of patients was 1,052 , and 1,021 patients respectively, while in the next 3 years the number decreased slightly - 922, 962 and 932 patients, respectively, which was followed by a novel increase in the number of patients in the last two years of the study $-1,019$ and 1,007 patients. As far as patients with chemical burns registered in the emergency room are concerned, there is a steady increase in their number, with a double amount of pa- tients recorded in 2018 compared to 2012.

TABLE 1. Number of patients with burns in period 2012-218

\begin{tabular}{|l|c|c|c|c|}
\hline Year & $\begin{array}{c}\text { EAU arrivals } \\
\text { chemical } \\
\text { burns }\end{array}$ & $\begin{array}{c}\text { Chemical } \\
\text { burns hos- } \\
\text { pitalisations }\end{array}$ & $\begin{array}{c}\text { Total burn } \\
\text { arrivals to } \\
\text { EAU }\end{array}$ & $\begin{array}{c}\text { Total burn } \\
\text { hospitalisa- } \\
\text { tions }\end{array}$ \\
\hline 2012 & 10 & 3 & 1,052 & 465 \\
\hline 2013 & 9 & 4 & 1,021 & 471 \\
\hline 2014 & 12 & 2 & 922 & 422 \\
\hline 2015 & 17 & 2 & 962 & 419 \\
\hline 2016 & 19 & 0 & 932 & 431 \\
\hline 2017 & 20 & 1 & 1,019 & 452 \\
\hline 2018 & 22 & 2 & 1,007 & 441 \\
\hline Total & 109 & 14 & 6,915 & 3,101 \\
\hline
\end{tabular}

The figure 3 shows the percentage of patients who have suffered chemical burns in the total number of patients who met the criteris for a burn diagnosis, upon arrival, with great accuracy. Thus, in 2012 the percentage of patients with chemical burns was $0.95 \%$ of all patients, in 2013 it was $0.88 \%$, this percentage increasing to $2.18 \%$ in 2018.

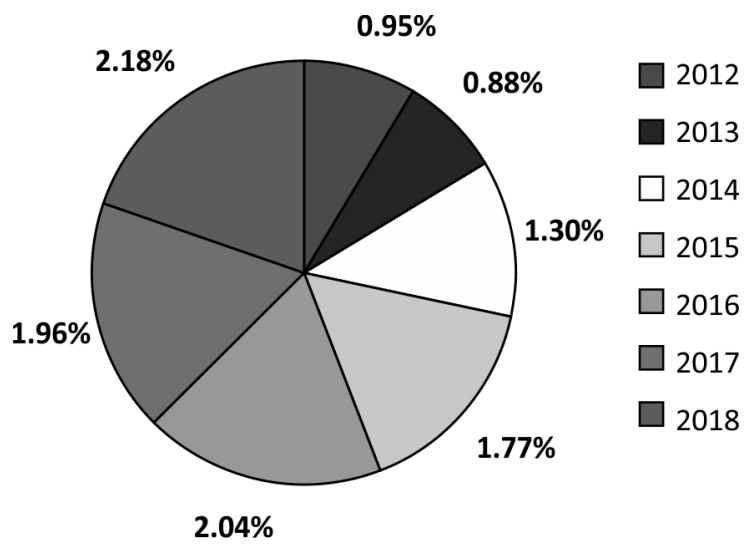

FIGURE 3. Annual distribution of cases arrived in the EAU for chemical burns versus the total number of arrivals for burns, during the period 2012-2018

Patients with chemical burns represent a small proportion of burn patients hospitalised in the Plastic Surgery and Burns ward of "Grigore Alexandrescu" Emergency Clinical Hospital. Over the course of the time period analysed within the study, namely 7 years, 14 children were hospitalised, most of whom were hospitalised during the first part of the study: $2012-3$ children, 2013 - 4 children, after which there was a decreasing trend in the number of hospitalised children with chemical burns: $2017-1$ child, $2018-2$ children, although the number of arrivals in the hospital's emergency room increased, in comparison with the onset of the study - Figure 4. 
$\square$ Chemical burns presenting to the ER

$\square$ Hospitalisation chemical burns

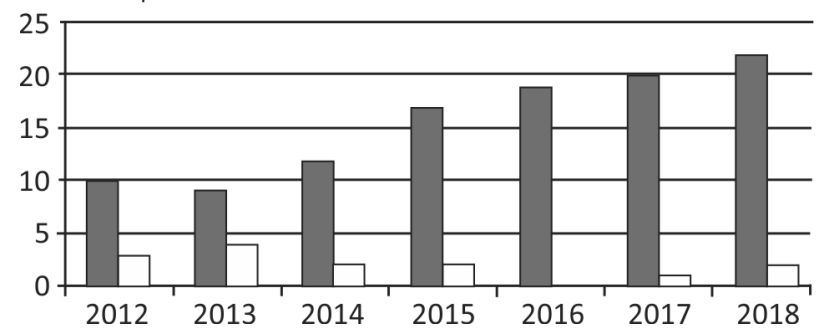

FIGURE 4. Annual evolution of patients with chemical burns, EAU visits, as well as those hospitalised

An analysis into the number of children arriving at the emergency room of "Grigore Alexandrescu" Clinical Emergency Hospital for Children between 2012 and 2018, as well as the number of patients hospitalised during the same period, reveals that the total number of arrivals of children with chemical burns has increased, in contrast to the number of those requiring hospitalization, which has decreased - Figure 5.

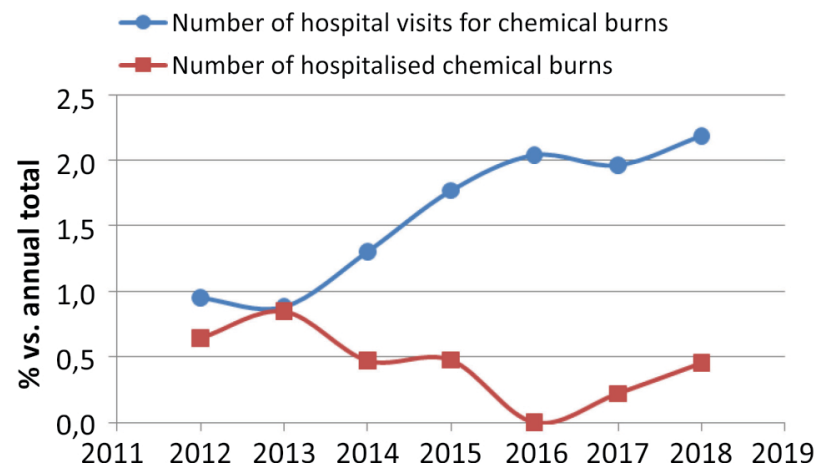

FIGURE 5. Evolution of hospitalised patients compared with EAU arrivals, based on the year of study

Multiple factors, such as the age of the patient, the location of the burn lesion, the concentration of the chemical substance which caused the lesion, the percentage of the affected body surface area, as well as the depth of the burn, are involved in the decision to hospitalise patients with chemical burns.

The body surface area affected by chemical burns presents a high degree of variation. 83 of the patients who arrived in the emergency room of "Grigore Alexandrescu" Clinical Emergency Hospital for Children, i.e. $78 \%$, have a percentage of the affected body surface area of less than $5 \%$, while the remaining 23 patients, $22 \%$, have burn injuries on an area of more than $5 \%$. The data in the study initially amounted to an average value of the affected body area index of 3,1, while dropping to the average value of the affected body area index of 2.6 in the last year of study, which can be translated into figures as follows: in 2012-2013 there were 3 , respectively 4 patients with burns affecting a body area of more than $5 \%$ of the total of 10 and
9 patients respectively, while in 2018, there were 5 patients with a larger body area affected, out of a total of 22 patients.

The depth of the burns is an important factor to consider over the course of the study period, and it should be brought to attention that in 2012 there were 5 patients with IIA-IIB and IIB-III degree burns out of a total of 10 patients who arrived in the hospital emergency room, i.e. 50\%, and in 2018, there were 9 patients out of the total of 22 patients in the emergency room, i.e. $40 \%$. It is therefore visible that there is a decrease in the depth of the chemical burns present in most patients with chemical burns who were examined.

We analysed the environment in which the chemical burn occurred as well: in the family, domestic, environment, in which the child is familiar and in theory, much better supervised by the parents, or outside the household, in the playgrounds, where the supervision of minors, especially those of older age is deficient Figure 6.

It is found that the vast majority of burns, approximately $70 \%$, occur at home, while the child is under the direct supervision of the parents.

Burns occuring in a $\square$ domestic / $\square$ external environment

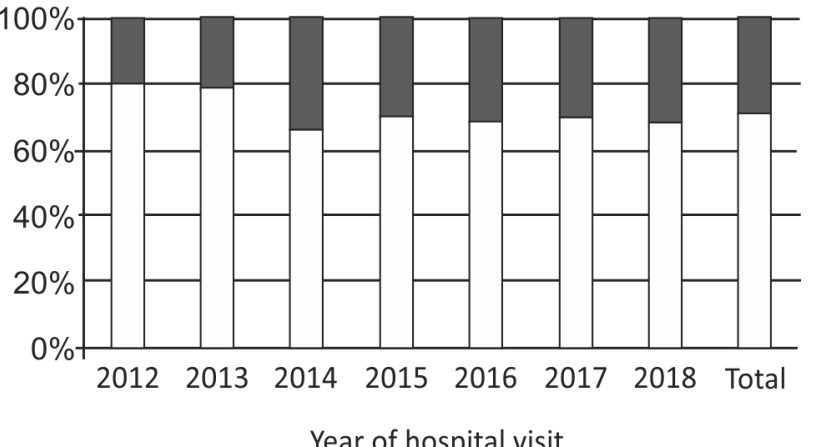

FIGURE 6. Annual distribution of patients with chemical burns who arrived in the EAU, according to the environment where the burn occurred

In the first years of the study, the percentage of patients requiring hospitalisation represented $30-45 \%$ of all patients who arrived in the emergency room, while by the end of the study period their percentage decreased, with hospitalisations accounting for only $10 \%$.

The figure 7 shows the annual trend regarding the number of hospitalisations of patients with chemical burns, which is slightly decreasing, albeit at a statistically insignificant rate.

\section{DISCUSSION}

Unfortunately, the percentage of children with burns accounts for a large proportion of the total num- 
Hospitalisation vs hospital visit - patients with chemical burns

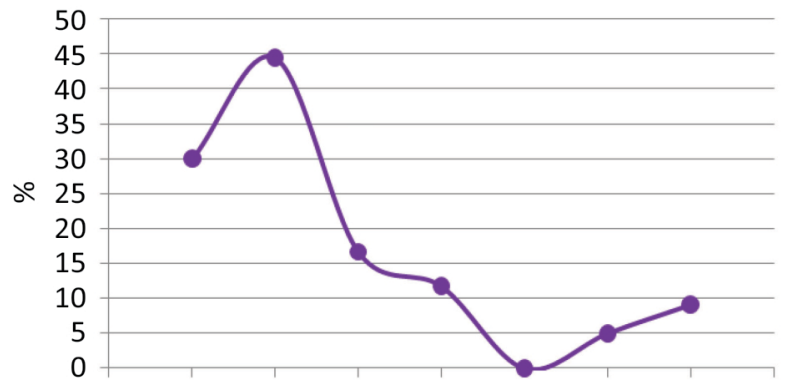

201120122013201420152016201720182019

FIGURE 7. Distribution of patients with chemical burns who are hospitalised in comparison with the visits to the EAU, based on the year within the study

ber of patients with burns worldwide. For example, studies conducted in southeast China during the years 2011-2015 indicate that the percentage of children who suffered burns amounts to approximately $39 \%$ of the total number of patients with burns treated in this region, although that very same percentage showed a gradual decrease over the course of the study (8). In Romania, at "Grigore Alexandrescu" Emergency Clinical Hospital, the total number of children with burns who arrived at the emergency room, in the initial phase of the study, was 1,052 patients, in 2012, while over the course of the following years, the number followed a downward trend, reaching the total of 1,007 children with burns, in 2018.

The majority of chemical burns occur in adults, in the industrial environment, being produced by high-concentration chemicals, which cause serious injuries. The vast majority of chemical burns in children occurr within the family environment, involving low-concentration chemicals, which accounts for the fact that the burns affecting children generally have a lower depth, in comparison with the burns produced by high-concentration chemicals.

As a result of prevention programmes implemented on a national level in recent years, the reduced access of minors to chemical substances, determines a decreased number of chemical burns produced in children, in comparison with burns of a distinct aetiology. The study conducted in the Sichuan Province of China shows that in a sample amounting to 1,387 children admitted with burn injuries, only 4 patients suffered chemical burns, which translates to a percentage of 0.28 of all children hospitalised with burns, over 7 years, 2003-2009 (9). In our study, the total number of children hospitalised with burns was 3,101 patients, with 14 cases of chemical burns, with a percentual value of $0.45 \%$ ot the total sample. The number of chemical burns encountered in children is very low, both in our country, as well as worldwide. According to the study conducted in Iran by Alagehbandan R., the percentage of chemical burns in children is $0.5 \%$, this type of injury being rare amongst all centers which treat burns (10).

During the timeframe that the study was conducted in, the number of patients with chemical burns who arrived at "Grigore Alexandrescu" Emergency Admission Unit of the Emergency Clinical Hospital increased. In 2012, there were 10 patients, while in 2018, there were 22 patients. The fact that the number of children with chemical burns has increased, even if they did not require hospitalization, raises concern and draws attention to the prevention measures applied at a national level. The percentage of chemical burns occuring in the household, while children are supervised by their family, is generally around $70 \%$. The same increased percentage of burns in the family environment is observed in other studies, conducted in countries such as Iran, or China $(9,11)$. At a young age, it is the role of parents to protect their offsprings from harmful chemicals, while at school there is clear need for educational programmes, which teach children to avoid these substances at all costs, and they should also be presented with the harmful effects which certain aetiological agents produce. On an industry level, international regulations regarding the concentration of chemical substances which are in extensive use, by the general population, as well as the safety measures applied to these products - for example the cap must not be easily unfastened - must be respected. Governments must be actively involved in monitoring compliance with these prevention measures regarding chemical burns (11).

The information regarding the percentage of body surface area affected describes a downward curve of this variable, starting from a maximum value of $4.44 \%$ during the first years of the study, and reaching an average value of about $2.41 \%$, at the end of the study period, in 2018. However, compared to other studies analysing the same variables, where the average value of the affected area is of approximately $1 \%(6)$, in our country, the area affected by the burn is much larger, therefore indicating a need to rethink national programmes regarding burns.

An additional variable analysed, namely the depth of the burn, shows that here too, the burns display a downward trajectory. The year 2018 brings us a lower percentage of patients who suffered deep burns $40 \%$, compared to the initial percentage of patients in 2012, 50\%. The information provided by the medical documents reveals the fact that patients with chemical burns examined over the period between 2012 and 2018 increased in number each year, while the number of those requiring hospitalisation decreased -14 patients over 7 years, which is gratifying, taking into consideration the fact that chemical burns have a high mortality compared to other types of burns, due to the possibility of systemic intoxication (7). 


\section{CONCLUSIONS}

The vast majority of pediatric burns can be prevented and require society as a whole to have common ground, and to take measures, in order to avoid these public health problems. Prevention measures in the case of burns must be supported by improving liv- ing conditions, providing protective equipment and gear against hot water and electricity, as well as the adequate regulation of chemical products.

Chemical burns account for a small percentage of the total number of burns, but they do require special attention and adequate management, due to the important economic and emotional impact they yield.

Conflict of interest: none declared Financial support: none declared

\section{REFERENCES}

1. Enescu M. Dan, Enescu Mihaela. Arsurile copilului. Bucureşti, Editura MedicArt, 2003.

2. Lascar I, Bordeianu I, Bratu T, DumitrescuIonescu D, Enescu D, Florescu PI, Georgescu D, Matusz P, Mugea T, Stamate T. Principii de chirurgie plastică şi microchirurgie reconstructivă. Bucureşti: Colecţia Monografii Medicale, Editura Naţional, 2005.

3. Enescu DM, Bordeianu I. Manual de chirurgie plastică. Ovidius University Press, 2001.

4. Herndon DN (red.). Total Burn Care, Ilth and Illth editions. Saunders Elsevier; 1999, 2007.
5. Palao R, Monge I, Ruiz M, Barret JP Chemical burns: Pathophysiology and treatment. Burns. 2010;36(3):295-304.

6. D'Cruz R, Pang TC, Harvey JG, Holland AJ. Chemical burns in children: Aetiology and prevention. Burns. 2015;41(4):764-769.

7. Hardwicke J, Hunter T, Staruch R, Moiemen N. Chemical burns - an historical comparison and review of the literature. Burns. 2012;38(3):383-387.

8. Li H, Wang S, Tan J, Zhou J, Wu J, Luo G. Epidemiology of pediatric burns in southwest China from 2011 to 2015. Burns. 2017:43(6):1306-1317.
9. Liu Y, Cen Y, Chen JJ, Xu XW, Liu XX Characteristics of paediatric burns in Sichuan province: epidemiology and prevention. Burns. 2012;38(1):26-31.

10. Alaghehbandan R, MacKay Rossignol A, Rastegar Lari A. Pediatric burn injuries in Tehran, Iran. Burns. 2001;27(2):115-118.

11. Kai-Yang L, Zhao-Fan X, Luo-Man Z et al. Epidemiology of pediatric burns requiring hospitalization in China: A literature review of retrospective studies. Pediatrics. 2008;122(1):132-142. 